\title{
The role of structural variants in pest adaptation and genome evolution of the Colorado potato beetle, Leptinotarsa decemlineata (Say)
}

\author{
Zachary Cohen ${ }^{1}$, D J Hawthorne ${ }^{2}$, and Sean Schoville ${ }^{1}$ \\ ${ }^{1}$ University of Wisconsin Madison \\ ${ }^{2}$ University of Maryland
}

September 8, 2021

\begin{abstract}
Structural variations (SVs) have been associated with genetic diversity and adaptation in diverse taxa. Despite these observations, it is not yet clear what their relative importance is for microevolution, especially with respect to known drivers of diversity, e.g., nucleotide substitutions, in rapidly adapting species. Here we examine the significance of SVs in pesticide resistance evolution of the agricultural super-pest, the Colorado potato beetle, Leptinotarsa decemlineata. By employing a trio-binning procedure, we develop near chromosomal reference genomes to characterize structural variation within this species. These updated assemblies represent $>100$-fold improvement of contiguity and include derived pest and ancestral non-pest individuals. We identify $>200,000 \mathrm{SVs}$, which appear to be non-randomly distributed across the genome as they co-occur with transposable elements. SVs intersect exons for genes associated with insecticide resistance, development, and transcription, most notably cytochrome P450 (CYP) genes. To understand the role that SVs might play in adaptation, we incorporate an additional 66 genomes among pest and non-pest populations of North America into the SV graph. Single nucleotide polymorphisms (SNPs) and SVs have a similar proportion in coding and non-coding regions of the genome, but there is a deficit of SNPs in SVs, suggesting SVs may be under selection. Using multiple lines of evidence, we identify 28 positively selected genes that include $337 \mathrm{SVs}$ and 442 outlier SNPs. Among these, there are four associated with insecticide resistance. Two of these genes (CYP4g15 and glycosyltransferase-13) are physically linked by a structural variant and have previously been shown to be co-induced during insecticide exposure.
\end{abstract}

\section{Hosted file}

SV_728.docx available at https://authorea.com/users/433157/articles/536593-the-role-ofstructural-variants-in-pest-adaptation-and-genome-evolution-of-the-colorado-potato-

beetle-leptinotarsa-decemlineata-say 